\title{
Hemodynamics derived from computational fluid dynamics based on magnetic resonance angiography is associated with functional outcomes in atherosclerotic middle cerebral artery stenosis
}

\author{
Jiahua Wu ${ }^{1,2 \#}$, Peng Wang ${ }^{1 \#}$, Leilei Zhou ${ }^{1}$, Danfeng Zhang ${ }^{1}$, Qian Chen ${ }^{1}$, Cunnan Mao ${ }^{1}$, Wen Su ${ }^{1}$, \\ Yingsong Huo ${ }^{1}$, Jin Peng ${ }^{3}$, Xindao Yin ${ }^{1}$, Guozhong Chen ${ }^{1}$ \\ ${ }^{1}$ Department of Radiology, Nanjing First Hospital, Nanjing Medical University, Nanjing, China; ${ }^{2}$ Department of Radiology, Zhongshan Hospital, \\ Fudan University, Shanghai, China; ${ }^{3}$ Intervention Department, Chenggong Hospital Affiliated to Xiamen University, Xiamen, China
}

Contributions: (I) Conception and design: J Wu, X Yin, G Chen; (II) Administrative support: X Yin, G Chen; (III) Provision of study materials or patients: All authors; (IV) Collection and assembly of data: All authors; (V) Data analysis and interpretation: J Wu, L Zhou, X Yin, G Chen; (VI) Manuscript writing: All authors; (VII) Final approval of manuscript: All authors.

"These authors contributed equally to this work.

Correspondence to: Xindao Yin; Guozhong Chen. Department of Radiology, Nanjing First Hospital, Nanjing Medical University, 68 Changle Road, Nanjing 210006, China. Email: y.163yy@163.com; paddychen@163.com.

Background: To investigate the relationship between fluid-attenuated inversion recovery (FLAIR) vascular hyperintensity (FVH), hemodynamics, and functional outcome in atherosclerotic middle cerebral artery (MCA) stenosis using a computational fluid dynamics (CFD) model based on magnetic resonance angiography (MRA), according to a modified Rankin Scale (mRS) at 3 months.

Methods: A total of 120 patients with 50-99\% atherosclerotic MCA stenosis were included. The training and internal validation groups were composed of 99 participants and 21 participants, respectively. Demographic, imaging data, and functional outcome (mRS at 3 months) were collected. Hemodynamic parameters were obtained from the CFD model. The FVH score was based on the number of territories where FVH is positive, according to the spatial distribution in the Alberta Stroke Program Early Computed Tomography Score (ASPECTS). The prediction models were constructed according to clinical and hemodynamic parameters using multivariate logistic analysis. The DeLong test compared areas under the curves (AUCs) of the models.

Results: The multivariable logistic regression analysis showed that the National Institute of Health Stroke Scale (NIHSS) at admission, hypertension, hyperlipidemia, the ratio of wall shear stress before treatment (WSSR before$_{\text {, }}$, and difference in the ratio of wall shear stress (WSSR) were independently associated with functional outcome (all $\mathrm{P}<0.05$ ). In the training group before treatment, the AUC of model 1a (only clinical variables) and $2 \mathrm{a}$ (clinical variables with addition of $\mathrm{WSSR}_{\text {before }}$ ) were 0.750 and 0.802 . After treatment, the AUC of model $1 \mathrm{~b}$ (only clinical variables) and $2 \mathrm{~b}$ (clinical variables with addition of difference in WSSR) were 0.815 and 0.883 , respectively. The AUC of models with hemodynamic parameters was significantly higher than the models based on clinical variables only (all $\mathrm{P}<0.05$, DeLong test). In the internal validation group before treatment, the AUC of the model (clinical variables) was 0.782 , and that of the model (clinical variables and $\mathrm{WSSR}_{\text {before}}$ ) was 0.800 . After treatment, the AUC of the model (clinical variables) was 0.833 , and that of the model (clinical variables and difference in WSSR) was 0.861 . There were no significant differences between the good and the poor functional outcome group concerning $\mathrm{FVH}_{\text {before }}$ scores before treatment $(0.30 \pm 0.81$ vs. $0.26 \pm 0.97 ; \mathrm{P}=0.321)$ and $\mathrm{FVH}_{\text {after }}$ scores after treatment $(0.08 \pm 0.39$ vs. $0.00 \pm 0.00$; $\mathrm{P}=0.244)$.

Conclusions: Hemodynamics was associated with functional outcomes in patients with ischemic stroke 
attributed to atherosclerotic MCA stenosis, while FVH was not. Hemodynamic parameters were of great importance in the prediction models.

Keywords: Middle cerebral artery (MCA); arterial stenosis; stroke; computational fluid dynamics (CFD); magnetic resonance angiography (MRA)

Submitted Mar 26, 2021. Accepted for publication Jul 23, 2021.

doi: 10.21037/qims-21-337

View this article at: https://dx.doi.org/10.21037/qims-21-337

\section{Introduction}

Intracranial atherosclerosis (ICAS) has been regarded as one of the predominant causes of acute ischemic stroke (AIS), which contributes to $30-50 \%$ of ischemic stroke in Asia (1). The aim of ICAS-related stroke treatment is to recanalize vessels and improve the reperfusion of ischemic brain parenchyma. Early functional outcome prediction is significant for ICAS-related stroke treatment. Good leptomeningeal collateral (LMC) status is associated with favorable outcomes of patients with ICAS steno-occlusive disease by sustaining the ischemic penumbra (2).

Previous studies have concluded that fluid-attenuated inversion recovery (FLAIR) vascular hyperintensity $(\mathrm{FVH})$ is related to large artery occlusion, representing slow retrograde flow in LMC (3). Previous studies have verified that both the FVH diffusion-weighted imaging (DWI) mismatch and FVH-Alberta Stroke Program Early Computed Tomography Score (ASPECTS) were associated with functional outcome and revascularization after receiving endovascular thrombectomy in acute stroke patients $(4,5)$. Compared with FVH-DWI mismatch, which is a qualitative approach, FVH-ASPECTS could quantify the degree of collateral circulation (6). Some studies have identified that high FVH scores before endovascular therapy predict a good functional outcome, while high FVH scores after endovascular therapy predict poor functional outcomes in acute stroke patients with large vessel stenosis and occlusion $(7,8)$.

The evolution of LMCs depends on a time-dependent process of arteriogenesis (9). Previous studies have shown that whole-brain and focal hemodynamics may play important roles in LMC evolution in ICAS-related stroke (10-12). A steep translesional pressure gradient can redistribute blood to the neighboring vascular networks in the condition of antegrade flow impedance in the main trunk. The resultant enhanced shear stress can promote collateral growth in the adjoining anastomosing arterioles promotes $(13,14)$. Computational fluid dynamics (CFD), as a computational flow simulation technique, has been applied to the study of associations between hemodynamics and atherosclerosis in the intracranial arteries $(10,11,15)$. Certain trends of longitudinal distribution of wall shear stress (WSS) and pressure have been found in ICAS stenosis (15). Leng et al. reported that a larger translesional pressure gradient was an independent predictor for good LMC in symptomatic middle cerebral artery (MCA) stenosis (10). Leng et al. further found that low translesional pressure ratio and high WSS ratio were independently associated with recurrent ischemic stroke in ICAS-related stroke (11). However, few studies based on CFD have investigated the association between hemodynamics and clinical outcome in patients with recent ischemic stroke attributed to atherosclerotic MCA stenosis.

Therefore, this study aimed to explore the potential associations between FVH, hemodynamic parameters derived from CFD, and clinical outcome in patients with recent ischemic stroke attributed to atherosclerotic MCA stenosis (50-99\%) respectively.

\section{Methods}

\section{Study design and participants}

This study was conducted in accordance with the Declaration of Helsinki (as revised in 2013). This was a retrospective cohort study approved by the Ethics Committee of the Nanjing Medical University. All participants or their legal representatives signed written informed consent. We analyzed consecutive acute stroke patients who were admitted to the Nanjing First Hospital between January 2018 and December 2020. Acute stroke was defined as an acute clinical vascular syndrome with cerebral infarction on DWI. Patients were enrolled based on the following inclusion criteria: (I) acute stroke patients at less than 4.5 hours of symptom onset; (II) ischemic stroke 
attributed to atherosclerotic MCA stenosis (50-99\%); (III) patients underwent clinically indicated computed tomography (CT) scanning and pretreatment multimodal magnetic resonance imaging (MRI) including FLAIR and contrast-enhanced magnetic resonance angiography (CEMRA). The exclusion criteria included: (I) inadequate image quality of MRA for CFD model; (II) any motion artifact of MRI; (III) any loss of clinical follow-up modified Rankin Scale (mRS) at 3 months; (IV) any intracranial tumor or trauma, arteriovenous malformation, or aneurysm. Demographics, National Institute of Health Stroke Scale (NIHSS) score, admission examination, medical history, and results of laboratory tests during hospitalization were collected. Functional outcome was evaluated based on the $\mathrm{mRS}$ at 3 months. Good functional outcome was defined as an $\mathrm{mRS}$ score $\leq 2$, otherwise $\geq 3$.

\section{MRI protocol}

All MRI scans, including pre-treatment and post-treatment, were performed on a 3.0 Tesla MRI scanner (Ingenia, Philips Medical Systems, Amsterdam, Netherlands) with an 8-channel receiver array head coil. The MRI protocol contained DWI axial scanning, FLAIR axis sequencing, and CE-MRA. The scanning parameters were as follows: FLAIR was obtained using the inverse recovery (IR) sequence, repetition time (TR), 7,000 ms; echo time (TE), $120 \mathrm{~ms}$; matrix, 356×151; field of view (FOV), $230 \mathrm{~mm} \times$ $230 \mathrm{~mm}$; flip angle (FA), $90^{\circ}$; slices, 18; thickness, $6 \mathrm{~mm}$; and intersection gap, $1.3 \mathrm{~mm}$. The DWI was obtained using the spin echo (SE) sequence, TR, 2,501 ms; TE, $98 \mathrm{~ms}$; matrix, 152×122; 3 directions; FOV, $230 \mathrm{~mm} \times 230 \mathrm{~mm}$; FA, $90^{\circ}$; slices, 18 ; thickness, $6 \mathrm{~mm}$; and intersection gap, $1.3 \mathrm{~mm}$. The DWI was achieved with b-values of 0 and $1,000 \mathrm{~s} / \mathrm{mm}^{2}$. The CE-MRA scans were obtained using the fast field echo (FFE) sequence, TR, $4.9 \mathrm{~ms}$; TE, $1.82 \mathrm{~ms}$; matrix, 528×531; FOV, $330 \mathrm{~mm} \times 330 \mathrm{~mm}$; and thickness, $1.2 \mathrm{~mm}$.

\section{CFD modeling}

Firstly, the patient-specific models were reconstructed based on MRA images. In brief, 2D cross-sectional images were imported into the software Mimics version 16.0 (Materialize NV, Leuven, Belgium) for image segmentation and reconstruction. The patient-specific geometry included the terminal intracranial segment of the internal carotid artery (ICA), the main segment of the MCA, and the proximal anterior cerebral artery (ACA). Secondly, the procedure of mesh was computed in the program ICEM CFD version 15.0 (Ansys Inc., Canonsburg, PN, USA); mesh sizes varied between 500,000 and 900,000 elements. Thirdly, mesh files were imported into the program Fluent version 15.0 (Ansys Inc., Canonsburg, PN, USA), and unsteady CFD blood flow simulation was performed using a finite volume approach to solve the Navier-Stokes equations and continuity equation.

The boundary conditions and assumptions were applied as follows: (I) rigid vessel walls with no-slip flow conditions were assumed; (II) blood was assumed as an incompressible, viscous Newtonian fluid with a constant viscosity of $0.0035 \mathrm{~kg} / \mathrm{m} / \mathrm{s}$ and density of $1,056 \mathrm{~kg} / \mathrm{m}^{3}$; (III) the outlet pressure was set at each participant's mean arterial pressure at admission; (IV) the inlet condition was set at the pulsatile volume flow waveform of a healthy volunteer, based on phase contrast MRA. According to the inlet volume flow rate, the mean blood velocity at the inlet of ICA was set when the constant radius of the vessel was assumed.

\section{Definition and measurement of hemodynamic and morphologic parameters in CFD models}

All simulated CFD models were post-processed in CFDPost, version 15.0 (Ansys, Inc.), to extract and measure hemodynamic parameters. The parameters including pressure and WSS were measured on the CFD models. Relative changes of pressure and WSS were defined as the ratio of pressure $\left(\mathrm{PR}_{\text {before }}\right)$, WSS (WSSR $\left.\mathrm{Wefore}_{\text {ere }}\right)$ before treatment and pressure $\left(\mathrm{PR}_{\text {after }}\right)$, WSS (WSSR after $)$ after treatment. The PR was calculated as pressure poststenotic $^{\prime}$ pressure $_{\text {prestenotic }}$, with pressure poststenotic $_{\text {and }}$ pressure ${ }_{\text {prestenotic }}$ measured at the first anatomically normal diameter distal and proximal to the ICAS lesion, respectively. The WSSR was calculated as $\mathrm{WSS}_{\text {stenotic }} / \mathrm{WSS}_{\text {prestenotic }}$, with $\mathrm{WSS}_{\text {stenotic }}$ and $\mathrm{WSS}_{\text {prestenotic }}$ measured at the most severely stenotic cross section of the vessel and the first anatomically normal diameter proximal to the ICAS lesion, respectively. The pressure was measured by spherical volumes of interest (VOI) and the others were by cut-planes. All cut-planes mentioned above were perpendicular to the direction of blood flow. The cut-planes were at the same locations before and after treatment. Differences in PR and WSSR were the differences between pre-treatment and post-treatment of PR and WSSR. Difference in PR $=\mathrm{PR}_{\mathrm{after}}-\mathrm{PR}_{\text {before }}$; difference in WSSR $=\mathrm{WSSR}_{\text {before }}-$ WSSR $_{\text {after }}$. 


\section{FVH analysis}

The FVH was defined as focal, circular, or serpentine high signal intensity in the brain parenchyma or cortical surface connecting the subarachnoid space on FLAIR images, representing slow blood flow in LMCs and corresponding to the typical arterial course (16). The FVH score was based on the number of territories where FVH was positive, according to the spatial distribution in the ASPECTS cortical areas (including insula and M1-M6). The ASPECT collateral scoring is a validated method to assess both tissue status with either CT or MR imaging and vascular status (17). A total of 7 territories were defined in each hemisphere. The level of the basal ganglia included the insula and M1 to M3, and the level of the ventricles above the basal ganglia included the M4 to M6 (18). Each territory was recorded as 1 point, and FVH scores ranged from 0 to 7 . The FVH was independently assessed by two investigators (CGZ and $\mathrm{WJH})$. A consensus was reached via consultation if any disagreement arose between the two investigators regarding the FVH score. We defined FVH scores before treatment as $\mathrm{FVH}_{\text {before }}$ and $\mathrm{FVH}$ scores after treatment as $\mathrm{FVH}_{\text {after. }}$.

\section{Multivariable logistic regression analysis and definition of prediction models}

The consecutive patients were assigned into the training group (patients from January 2018 to June 2020) and the internal validation group (patients from July 2020 to December 2020). Multivariable logistic regression analysis was performed in the training group and applied to all internal validation groups.

Different multivariable models were constructed as follows. Before treatment: (I) model 1a was based on clinical variables (NIHSS at admission, hypertension, and hyperlipidemia); (II) model 2a was based on clinical variables and $W_{S S R}$ before. After treatment: (I) model $1 \mathrm{~b}$ was based on clinical variables (NIHSS at admission, hypertension, and hyperlipidemia); (II) model 2b was based on clinical variables and differences in WSSR.

\section{Statistical analysis}

All continuous variables were shown as the mean \pm standard deviation (SD), whereas categorical variables were presented as absolute and relative frequencies. Differences between groups were analyzed using the chi-squared test for categorical variables and an independent-samples t-test or Fisher's exact test for continuous variables. Nonparametric tests were used to analyze non-normally distributed data. Multivariable logistic regression analysis and receiver operating curve (ROC) analysis were used to assess the prognostic value of clinical variables and hemodynamics on functional outcome, and the odds ratio (OR) and 95\% confidence interval (CI) were obtained. The DeLong test was used to compare the differences of areas under the curves (AUCs). The inter-reader agreement was evaluated according to the Kappa analysis. The $\kappa$ value was between 0 and 1 . A $\kappa$ value less than 0.2 indicated poor agreement; the range between 0.2 and 0.4 indicated general agreement; the agreement between 0.4 and 0.6 was moderate; the $\kappa$ value of $0.6-0.8$ indicated good agreement; the $\kappa$ value of $0.8-1.0$ was excellent agreement. All tests were carried out using the software SPSS version 20.0 (IBM Corp., Armonk, NY, USA). Differences were considered statistically significant when $\mathrm{P}<0.05$.

\section{Results}

A total of 156 patients with recent ischemic stroke attributed to atherosclerotic MCA stenosis (50-99\%) were screened. Of these, 36 patients were excluded (severe artifacts on MRI, $n=24$; inadequate image quality of MRA for CFD model, $\mathrm{n}=12$ ); there were 120 patients included in the final analysis (mean age $69.40 \pm 10.85$ years; 72 males). The training and internal validation groups were composed of 99 participants and 21 participants, respectively, among whom 60 participants and 12 participants underwent multimodal MRI including FLAIR and CE-MRA within $48 \mathrm{~h}$ after treatment, respectively. Training group participants were divided into a good functional outcome group (mRS $=0-2, \mathrm{n}=60,60.61 \%$ ) and a poor functional outcome group ( $\mathrm{mRS}=3-6, \mathrm{n}=39,39.39 \%)$. The participants were divided into good functional outcome groups in the internal validation group $(\mathrm{mRS}=0-2, \mathrm{n}=10$, $47.62 \%$ ) and poor functional outcome group ( $\mathrm{mRS}=3-6$, $\mathrm{n}=11,52.38 \%)$.

Detailed demographic information of two functional outcome subgroups of the training group is shown in Table 1 . Compared with the good functional outcome group, more participants had a history of hypertension $(89.74 \%$ vs. $71.67 \% ; \mathrm{P}=0.032)$ and hyperlipidemia $(43.59 \%$ vs. $20 \% ; \mathrm{P}=0.012)$ in the poor functional outcome group. The NIHSS score at admission $(9.95 \pm 7.15$ vs. $6.65 \pm 4.85$; $\mathrm{P}=0.015)$ of the poor functional outcome group was 
Table 1 Characteristics of training group participants

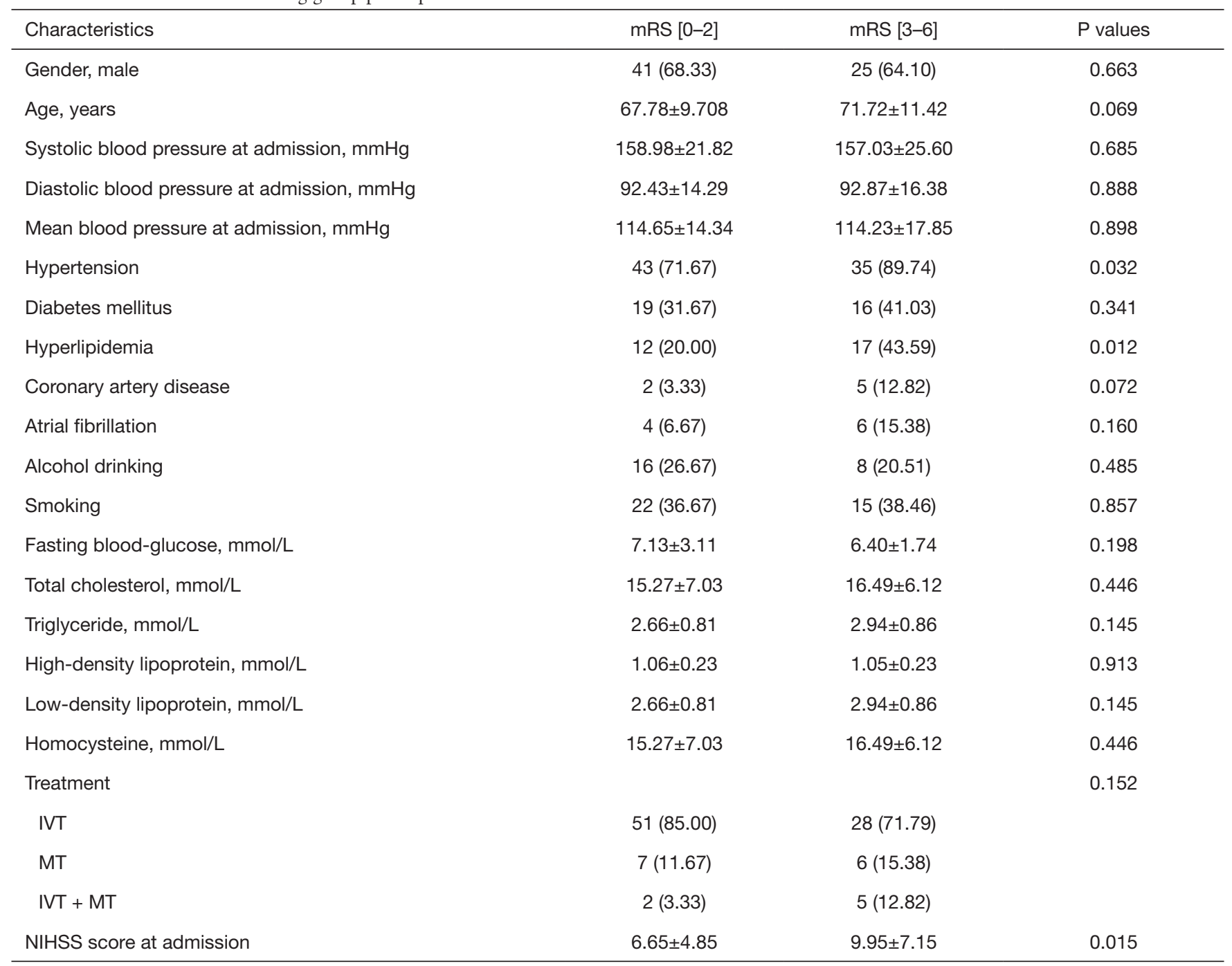

Continuous data are presented as the mean \pm SD, whereas categorical variables are shown as frequencies. Independent-samples $t$-tests were used for continuous variables and chi-squared tests were used for categorical variables. The nonparametric tests were used to analyze non-normally distributed data. mRS, modified Rankin Scale; IVT, intravenous thrombolysis; MT, mechanical thrombectomy; NIHSS, National Institute of Health Stroke Scale; SD, standard deviation.

significantly higher than that of the good functional outcome group. There were no statistically significant differences between two groups concerning age, gender, consumption of alcohol, smoking, results of laboratory tests or treatment (all $\mathrm{P}>0.05)$. In hemodynamic parameters before and after treatment, WSSR $_{\text {before }}(6.92 \pm 7.80$ vs. $3.75 \pm 3.13 ; \mathrm{P}=0.022)$, the difference in WSSR $(5.93 \pm 8.14$ vs. $1.50 \pm 2.09 ; \mathrm{P}=0.004)$ in the good functional outcome group were significantly higher than those in the poor functional outcome group. However, there were no statistical differences in $\mathrm{PR}_{\text {before }}(\mathrm{P}=0.229)$ and difference in $\mathrm{PR}(\mathrm{P}=0.271)$ between two groups (Table 2, Figures 1,2$)$. In FVH before and after treatment, the inter-reader agreement of assessing FVH was excellent, with a $\kappa$ value of 0.812 . There were no significant differences between the good and the poor functional outcome groups concerning $\mathrm{FVH}_{\text {before }}$ scores $(0.30 \pm 0.81$ vs. $0.26 \pm 0.97 ; \mathrm{P}=0.321)$ and $\mathrm{FVH}_{\text {after }}$ scores $(0.08 \pm 0.39$ vs. $0.00 \pm 0.00 ; \mathrm{P}=0.244)$ (Table 2, Figures 1,2).

The multivariable logistic regression analysis showed that NIHSS score at admission, hypertension, hyperlipidemia, WSSR $_{\text {before }}$, and difference in WSSR was independently 
Table 2 Characteristics of hemodynamic parameters and FVH before and after treatment

\begin{tabular}{lccc}
\hline Characteristics & mRS [0-2] & mRS [3-6] & P values \\
\hline WSSR $_{\text {before }}$ & $6.92 \pm 7.80$ & $3.75 \pm 3.13$ & 0.022 \\
PR $_{\text {before }}$ & $0.98 \pm 0.03$ & $0.99 \pm 0.01$ & 0.229 \\
Difference in WSSR & $5.93 \pm 8.14$ & $1.50 \pm 2.09$ & 0.004 \\
Difference in PR & $0.011 \pm 0.012$ & $0.010 \pm 0.014$ & 0.271 \\
$\mathrm{FVH}_{\text {before }}$ scores & $0.30 \pm 0.81$ & $0.26 \pm 0.97$ & 0.321 \\
$\mathrm{FVH}_{\text {after }}$ scores & $0.08 \pm 0.39$ & $0.00 \pm 0.00$ & 0.244
\end{tabular}

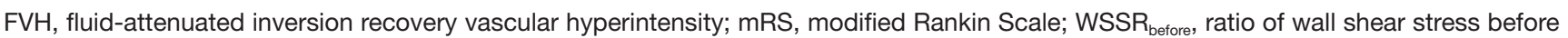
treatment; $\mathrm{PR}_{\text {before }}$, ratio of pressure before treatment; difference in WSSR and difference in PR were the difference between pre-treatment and post-treatment of WSSR and $\mathrm{PR} ; \mathrm{FVH}_{\text {before, }} \mathrm{FVH}$ before treatment; $\mathrm{FVH}_{\text {after, }}$ FVH after treatment.
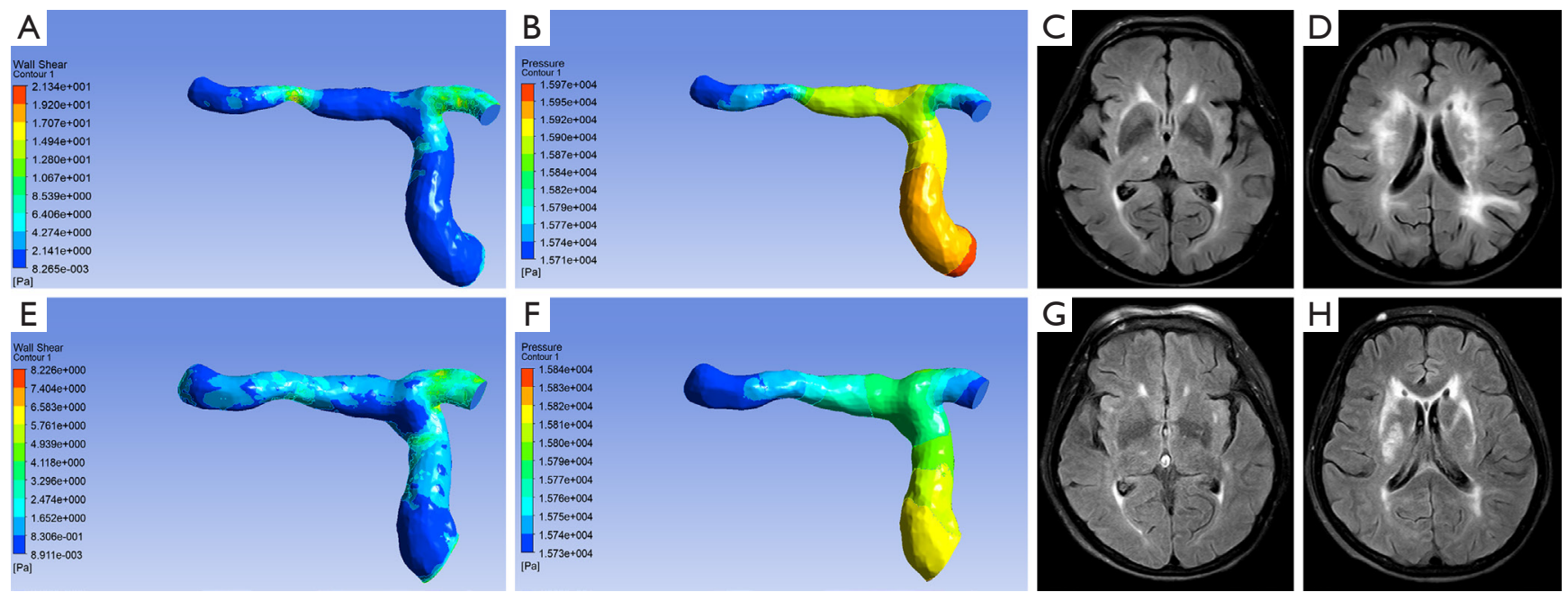

Figure 1 CFD models and FLAIR images for a case with atherosclerotic right MCA stenosis, who had good functional outcome (mRS at 3 months, 2 score). (A,B) Contours of WSS and pressure before treatment. WSSR of this case before treatment was 6.10 and PR was 0.99 . (C,D) FLAIR images before treatment with no FVH (FVH score $=0)$. (E,F) Contours of WSS and pressure after treatment. WSSR of this case after treatment was 1.49 and PR was 1.00. Difference in WSSR =4.61, Difference in PR =0.01. (G,H) FLAIR images after treatment with no FVH (FVH score =0). CFD, computational fluid dynamics; FLAIR, fluid-attenuated inversion recovery; MCA, middle cerebral artery; mRS, modified Rankin Scale; WSS, wall shear stress; WSSR, ratio of wall shear stress; PR, ratio of pressure; FVH, fluid-attenuated inversion recovery vascular hyperintensity.

associated with functional outcome (Tables 3,4). Before treatment, the AUC of the model 1a (clinical variables) and model $2 \mathrm{a}$ (clinical variables and $\mathrm{WSSR}_{\text {before }}$ ) were 0.750 (95\% CI: 0.650 to 0.851 ) and 0.802 (95\% CI: 0.714 to $0.890)$ respectively. After treatment, the AUC of the model $1 \mathrm{~b}$ (clinical variables) and model $2 \mathrm{~b}$ (clinical variables and difference in WSSR) were 0.815 (95\% CI: 0.705 to 0.925 ) and 0.883 (95\% CI: 0.798 to 0.968$)$ respectively. The AUCs of models with hemodynamic parameters were significantly higher than those of the models based on clinical variables (all $\mathrm{P}<0.05$, DeLong test) (Figure 3). In internal validation datasets before treatment, the AUC of model $1 \mathrm{~b}$ (clinical variables) was 0.782 (95\% CI: 0.553 to 1.000$)$ and that of model $2 \mathrm{~b}$ (clinical variables and $\mathrm{WSSR}_{\text {before }}$ ) was $0.800(95 \%$ CI: 0.605 to 0.995$)$. After treatment, the AUC of $1 \mathrm{~b}$ model was 0.833 (95\% CI: 0.590 to 1.000 ), and that of model $2 \mathrm{~b}$ (clinical variables and difference in WSSR) was $0.861(95 \%$ CI: 0.634 to 1.000$)$. 

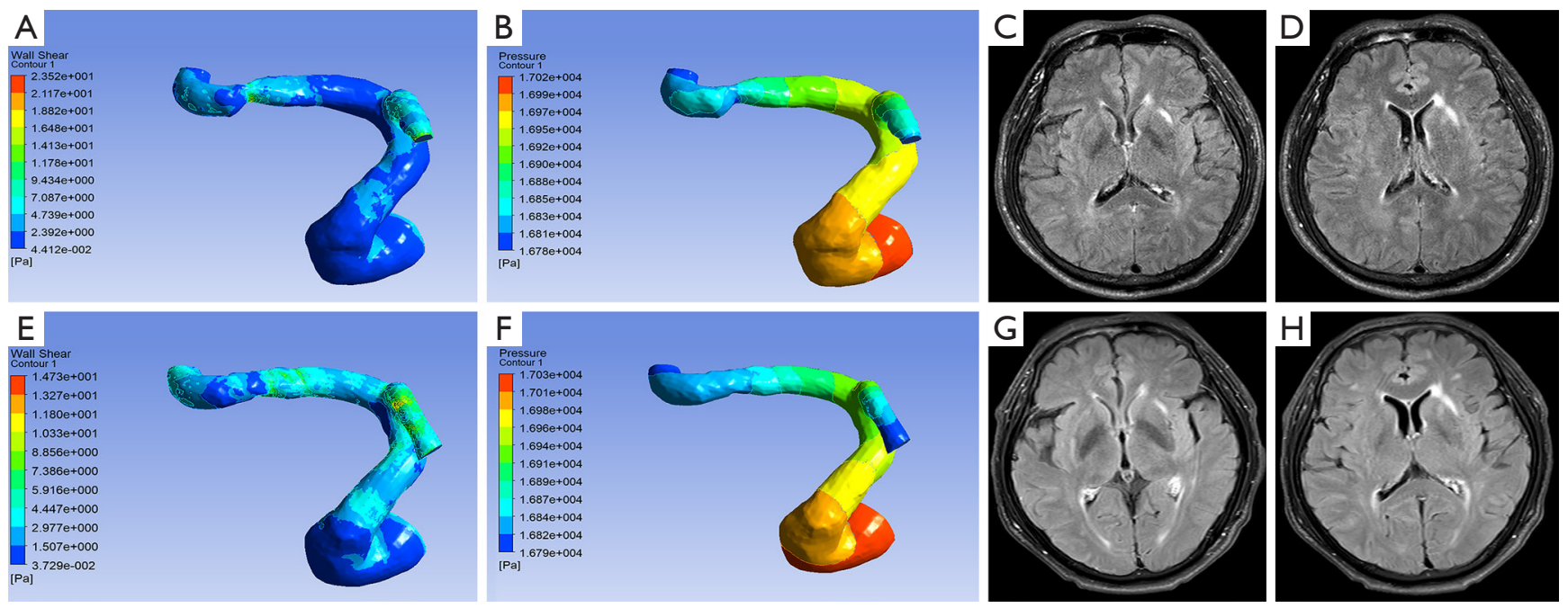

Figure 2 CFD models and FLAIR images for a case with atherosclerotic right MCA stenosis, who had poor functional outcome (mRS at 3 months, 3 score). (A,B) Contours of WSS and pressure before treatment. WSSR of this case before treatment was 2.78 and PR was 0.98. (C,D) FLAIR images before treatment with no FVH (FVH score $=0)$. (E,F) Contours of WSS and pressure after treatment. WSSR of this case after treatment was 1.27 and PR was 1.00. Difference in WSSR =1.51, Difference in PR =0.02. (G,H) FLAIR images after treatment with no FVH (FVH score =0). CFD, computational fluid dynamics; FLAIR, fluid-attenuated inversion recovery; MCA, middle cerebral artery; mRS, modified Rankin Scale; WSS, wall shear stress; WSSR, ratio of wall shear stress; PR, ratio of pressure; FVH, fluid-attenuated inversion recovery vascular hyperintensity.

Table 3 Two multivariable models before treatment in predicting functional outcome

\begin{tabular}{lcc}
\hline & & $95 \% \mathrm{Cl}$ for OR \\
\cline { 2 - 3 } Parameters & Model 1a & Model 2a \\
\hline NIHSS score at admission & $1.124^{\star}(1.037-1.218)$ & $1.124^{\star}(1.033-1.222)$ \\
Hypertension & $0.209^{\star}(0.059-0.732)$ & $0.189^{\star}(0.052-0.685)$ \\
Hyperlipidemia & $0.287^{\star}(0.109-0.755)$ & $0.286^{\star}(0.104-0.791)$ \\
WSSR $_{\text {before }}$ & - & $0.857^{\star}(0.746-0.985)$ \\
AUCs for 95\% Cl & $0.750(0.653-0.832)$ & $0.802(0.710-0.875)$ \\
P value of DeLong test & & 0.041
\end{tabular}

*, $\mathrm{P}<0.05$. Model 1a, only clinical variables; model 2a, model 1a with addition of WSSR before. $\mathrm{Cl}$, confidence interval; OR, odds ratio; NIHSS,

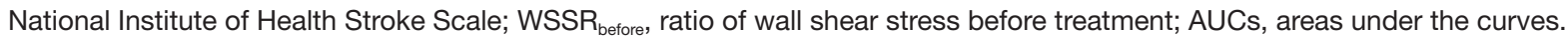

\section{Discussion}

In this study, we found that NIHSS score at admission, hypertension, hyperlipidemia, higher $\mathrm{WSSR}_{\text {before }}$, and higher difference in WSSR was independently associated with good functional outcome in patients with ischemic stroke attributed to atherosclerotic MCA stenosis. Furthermore, the amalgamation of hemodynamic parameters into a model for functional outcome prediction had a better performance than the model based on clinical characteristics. We also found that FVH before and after treatment showed no statistical association with functional outcome in patients with atherosclerotic MCA stenosis.

Previous studies $(7,19-22)$ have indicated that NIHSS score at admission, hypertension, and hyperlipidemia was associated with poor functional outcome, according to our study. Previous studies showed that people with diabetes had a higher chance of stroke and high death rates than those without diabetes $(23,24)$; however, our study showed 
Table 4 Two multivariable models after treatment in predicting functional outcome

\begin{tabular}{lcc}
\hline \multirow{2}{*}{ Parameters } & \multicolumn{2}{c}{$95 \%$ Cl for OR } \\
\cline { 2 - 3 } NIHSS score at admission & $1.261^{*}(1.069-1.488)$ & Model 2b \\
Hypertension & $0.213^{*}(0.046-0.980)$ & $0.138^{*}(0.026-0.746)$ \\
Hyperlipidemia & $0.178^{*}(0.048-0.665)$ & $0.145^{\star}(0.032-0.659)$ \\
Difference in WSSR & - & $0.723^{\star}(0.521-1.003)$ \\
AUCs for 95\% Cl & $0.815(0.694-0.904)$ & $0.883(0.774-0.952)$ \\
P value of DeLong test & & 0.043 \\
\hline
\end{tabular}

*, $\mathrm{P}<0.05$. Model $1 \mathrm{~b}$, only clinical variables; model $2 \mathrm{~b}$, model $1 \mathrm{~b}$ with addition of difference in WSSR. Cl, confidence interval; OR, odds ratio; NIHSS, National Institute of Health Stroke Scale; difference in WSSR, the difference between pre-treatment and post-treatment of WSSR; AUCs, areas under the curves.
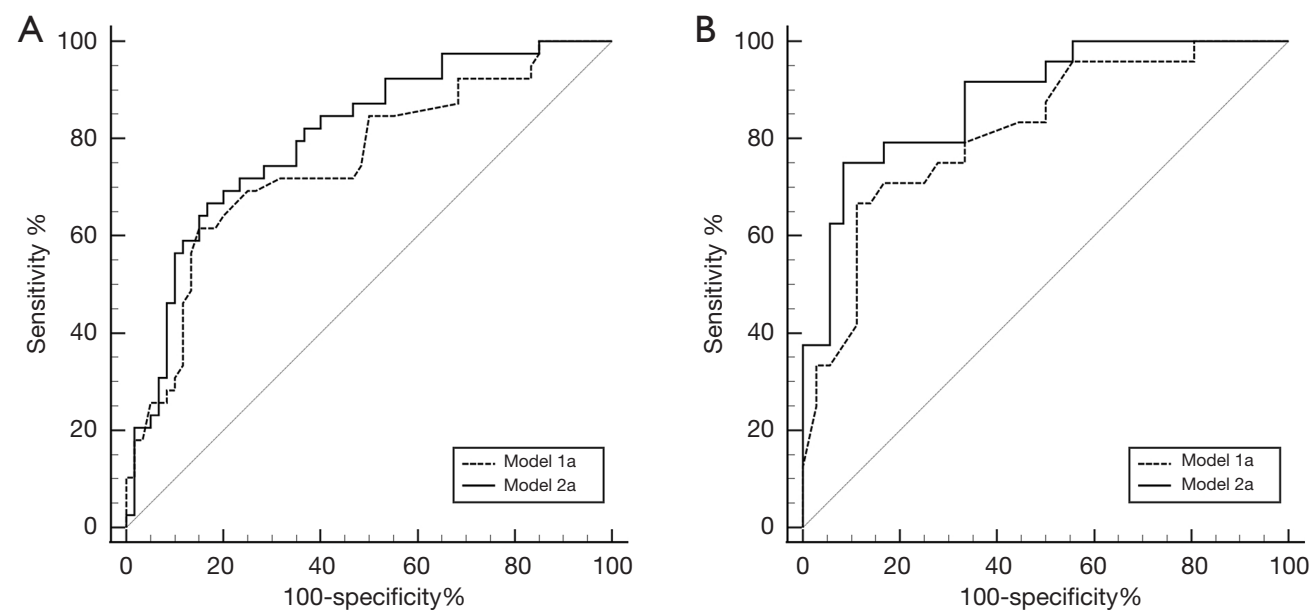

Figure 3 ROC curves of the multivariable models before and after treatment. (A) ROC curves of the multivariable models before treatment. Model 1a, only clinical variables; Model 2a, clinical variables with addition of $W_{S S R}$ before. The AUC of the model 1a and model $2 \mathrm{a}$ were 0.750 and 0.802 respectively. (B) ROC curves of the multivariable models after treatment. Model $1 \mathrm{~b}$, only clinical variables; model $2 \mathrm{~b}$, clinical variables with addition of difference in WSSR. The AUC of the model $1 \mathrm{~b}$ and model $2 \mathrm{~b}$ were 0.815 and 0.883 respectively. ROC, receiver operating characteristic; $W_{S S R}$ before, ratio of wall shear stress before treatment; AUC, area under the ROC curve; difference in WSSR, the difference between pre-treatment and post-treatment of WSSR.

no statistical association between diabetes and functional outcome. This may be due to our small sample size and inclusion of heterogeneous stroke subtypes.

Our study showed that low $\mathrm{WSSR}_{\text {before }}$ and difference in WSSR were associated with poor functional outcomes. The WSS rate was directly proportional to blood flow velocity and represents the spatial gradient or changes of blood flow velocity (25), which implied low $\mathrm{WSSR}_{\text {before }}$ and difference in WSSR might accompany slow velocity of blood flow before and after treatment. Low WSS could accelerate transportation of atherogenic matters to atherogenesis and promote plaque development and progression to atherosclerotic plaques with a large lipid core and thin fibrous cap and constrictive remodeling in a swine diabetic model of atherosclerosis $(26,27)$. The study based on an animal model illustrated that regions with low and oscillatory WSS were correlated with up regulation of pro-atherosclerotic genes, downregulation of antiatherosclerotic genes, endothelial dysfunction, and rapid atherosclerosis development. Moreover, low WSS vessel 
segments were more likely to undergo constrictive vascular remodeling (28). Therefore, low WSS upon the ICAS lesion may result in poor functional outcomes through mechanisms of atherosclerotic plaques development and constrictive vascular remodeling.

Further studies in vivo and in vitro are needed to elucidate the associations between WSS of ICAS and functional outcome. We detected a trend that lower PR might be related to good functional outcome, although no statistical association was found between PR and functional outcome, resulting from the potential influence of luminal stenosis degree (10). Leng et al. showed that a larger translesional pressure gradient (namely, a low translesional PR in this study) in severe MCA stenosis (70-99\%) was an essential force in good LMC status (10). The possible explanation was that a steep translesional pressure gradient forced blood flow into the pre-existing collateral anastomoses, which improved functional outcomes. Of note, in this study, the prediction performance was further improved when the hemodynamic parameters before and after treatment were integrated into the model based on clinical variables, which indicated the additional value of hemodynamics in predicting functional outcomes. Therefore, Hemodynamic parameters may be used as individual monitoring indicators to predict the functional outcome and guide the treatment. Future studies with larger sample sizes are warranted to validate the potential additional value of hemodynamics.

The FVH was described as hyperintensity in the parenchyma of the brain or at the cortical surface connecting the subarachnoid space, representing disordered or sluggish blood flow in collaterals (16), which was frequently found in large vessel steno-occlusive disease resulting from atherosclerosis $(7,29)$. Jiang et al. demonstrated that FVH before and after endovascular therapy was independently associated with functional outcomes in acute stroke patients with intracranial large artery occlusion (7). Previous studies also have clarified that the presence of FVH corresponded to proximal large vessel occlusion, particularly the proximal segment of the MCA (30). However, our results showed no statistical association between FVH and functional outcome in acute stroke patients with atherosclerotic MCA stenosis. The evolution of collaterals was a time-dependent process depending on arteriogenesis, involving the formation of blood vessels from pre-existing arterioles $(9,13)$. Moreover, compared with intracranial large artery occlusion, there was still residual blood flow through the stenotic vessels, which might affect the formation of collaterals and observation of $\mathrm{FVH}$.

Some limitations in the current study must be acknowledged. Firstly, this study was a retrospective singlecenter study, and the sample size was small. A prospective multi-centered study with a larger sample size needs to be conducted in the future. Secondly, a simplified CFD model was applied to assess hemodynamics of ICAS lesions, such as blood properties (rigid vessel walls, Newtonian fluid, and so on) and general boundary conditions (the inlet and outlet condition). Thirdly, the timing of the MRI after treatment within $24 \mathrm{~h}$ (51 participants) and in $48 \mathrm{~h}$ (21 participants) was ambiguous, which might have influenced the results because of the time dependence of FVH. Further details of the time dependence of FVH need to be investigated in the future. Fourthly, the degree of luminal stenosis was not dichotomized to moderate (50-69\%) and severe (70-99\%) stenosis according to the Warfarin-Aspirin Symptomatic Intracranial Disease (WASID) method (31,32), which might affect the results of hemodynamics. All factors mentioned above should be considered and efforts made to establish more accurate blood flow simulations in future studies.

\section{Conclusions}

In conclusion, WSSR $\mathrm{before}_{\text {and }}$ andference in WSSR were independently associated with functional outcome, while FVH before and after treatment showed no statistical association with functional outcome in patients with ischemic stroke attributed to atherosclerotic MCA stenosis. Moreover, the addition of hemodynamic parameters can improve the predictive ability of functional outcome, which implied the potential values of hemodynamic parameters based on CFD in clinical treatment for ICAS-related stroke. More prospective studies with larger sample sizes are needed to validate these findings.

\section{Acknowledgments}

Funding: This work was supported by the National Natural Science Foundation of China (82001811).

\section{Footnote}

Conflicts of Interest: All authors have completed the ICMJE uniform disclosure form (available at https://dx.doi. org/10.21037/qims-21-337). The authors have no conflicts of interest to declare.

Ethical Statement: The authors are accountable for all aspects of the work in ensuring that questions related 
to the accuracy or integrity of any part of the work are appropriately investigated and resolved. The study was conducted in accordance with the Declaration of Helsinki (as revised in 2013). This was a retrospective cohort study approved by the Ethics Committee of the Nanjing Medical University. All participants or their legal representatives signed a written informed consent.

Open Access Statement: This is an Open Access article distributed in accordance with the Creative Commons Attribution-NonCommercial-NoDerivs 4.0 International License (CC BY-NC-ND 4.0), which permits the noncommercial replication and distribution of the article with the strict proviso that no changes or edits are made and the original work is properly cited (including links to both the formal publication through the relevant DOI and the license). See: https://creativecommons.org/licenses/by-nc-nd/4.0/.

\section{References}

1. Wang Y, Zhao X, Liu L, Soo YO, Pu Y, Pan Y, Wang Y, Zou X, Leung TW, Cai Y, Bai Q, Wu Y, Wang C, Pan X, Luo B, Wong KS; CICAS Study Group. Prevalence and outcomes of symptomatic intracranial large artery stenoses and occlusions in China: the Chinese Intracranial Atherosclerosis (CICAS) Study. Stroke 2014;45:663-9.

2. Bang OY, Goyal M, Liebeskind DS. Collateral circulation in ischemic stroke: assessment tools and therapeutic strategies. Stroke 2015;46:3302-9.

3. Kufner A, Galinovic I, Ambrosi V, Nolte CH, Endres M, Fiebach JB, Ebinger M. Hyperintense vessels on FLAIR: hemodynamic correlates and response to thrombolysis. AJNR Am J Neuroradiol 2015;36:1426-30.

4. Legrand L, Turc G, Edjlali M, Beaumont M, Gautheron V, Ben Hassen W, Charron S, Trystram D, Boulouis G, Bourcier R, Benzakoun J, Naggara O, Clarençon F, Bracard S, Oppenheim C; THRACE Investigators. Benefit from revascularization after thrombectomy according to FLAIR vascular hyperintensities-DWI mismatch. Eur Radiol 2019;29:5567-76.

5. Jiang L, Peng M, Chen H, Geng W, Zhao B, Yin X, Chen YC, Su H. Diffusion-weighted imaging (DWI) ischemic volume is related to FLAIR hyperintensity-DWI mismatch and functional outcome after endovascular therapy. Quant Imaging Med Surg 2020;10:356-67.

6. Song L, Lyu C, Shen G, Guo T, Wang J, Wang W, Qiu X, Lerner A, Wintermark M, Gao B. Application of FLAIR vascular hyperintensity-DWI mismatch in ischemic stroke depending on semi-quantitative DWI-alberta stroke program early CT score. Front Neurol 2019;10:994.

7. Jiang L, Chen YC, Zhang H, Peng M, Chen H, Geng W, Xu Q, Yin X, Ma Y. FLAIR vascular hyperintensity in acute stroke is associated with collateralization and functional outcome. Eur Radiol 2019;29:4879-88.

8. Zhu L, Gong S, Zhu X, Zhang R, Ren K, Zhu Z, Wang T, Xing W. FLAIR vascular hyperintensity: an unfavorable marker of early neurological deterioration and short-term prognosis in acute ischemic stroke patients. Ann Palliat Med 2020;9:3144-51.

9. Scholz D, Cai WJ, Schaper W. Arteriogenesis, a new concept of vascular adaptation in occlusive disease. Angiogenesis 2001;4:247-57.

10. Leng X, Lan L, Ip HL, Fan F, Ma SH, Ma K, Liu H, Yan Z, Liu J, Abrigo J, Soo YOY, Liebeskind DS, Wong KS, Leung TW. Translesional pressure gradient and leptomeningeal collateral status in symptomatic middle cerebral artery stenosis. Eur J Neurol 2018;25:404-10.

11. Leng X, Lan L, Ip HL, Abrigo J, Scalzo F, Liu H, et al. Hemodynamics and stroke risk in intracranial atherosclerotic disease. Ann Neurol 2019;85:752-64.

12. Liebeskind DS, Cotsonis GA, Saver JL, Lynn MJ, Turan TN, Cloft HJ, Chimowitz MI; Warfarin-Aspirin Symptomatic Intracranial Disease (WASID) Investigators. Collaterals dramatically alter stroke risk in intracranial atherosclerosis. Ann Neurol 2011;69:963-74.

13. Buschmann I, Schaper W. The pathophysiology of the collateral circulation (arteriogenesis). J Pathol 2000;190:338-42.

14. Heil M, Schaper W. Pathophysiology of collateral development. Coron Artery Dis 2004;15:373-8.

15. Chen Z, Qin H, Liu J, Wu B, Cheng Z, Jiang Y, Liu L, Jing L, Leng X, Jing J, Wang Y, Wang Y. Characteristics of wall shear stress and pressure of intracranial atherosclerosis analyzed by a computational fluid dynamics model: a pilot study. Front Neurol 2019;10:1372.

16. Azizyan A, Sanossian N, Mogensen MA, Liebeskind DS. Fluid-attenuated inversion recovery vascular hyperintensities: an important imaging marker for cerebrovascular disease. AJNR Am J Neuroradiol 2011;32:1771-5.

17. Hohenhaus M, Schmidt WU, Brunecker P, Xu C, Hotter B, Rozanski M, Fiebach JB, Jungehülsing GJ. FLAIR vascular hyperintensities in acute ICA and MCA infarction: a marker for mismatch and stroke severity?. Cerebrovasc Dis 2012;34:63-9.

18. Li G, Huang R, Bi G. The impact of FLAIR vascular 
hyperintensity on clinical severity and outcome: a retrospective study in stroke patients with proximal middle cerebral artery stenosis or occlusion. Neurol Sci 2021;42:589-98.

19. Vangen-Lønne AM, Wilsgaard T, Johnsen SH, Løchen ML, Njølstad I, Mathiesen EB. Declining incidence of ischemic stroke: what is the impact of changing risk factors? The Tromsø Study 1995 to 2012. Stroke 2017;48:544-50.

20. Brooks DC, Schindler JL. Management of hyperlipidemia after stroke. Curr Treat Options Cardiovasc Med 2019;21:93.

21. Miao J, Zang X, Cui X, Zhang J. Autophagy, hyperlipidemia, and atherosclerosis. Adv Exp Med Biol 2020;1207:237-64.

22. Zechariah A, ElAli A, Hagemann N, Jin F, Doeppner TR, Helfrich I, Mies G, Hermann DM. Hyperlipidemia attenuates vascular endothelial growth factor-induced angiogenesis, impairs cerebral blood flow, and disturbs stroke recovery via decreased pericyte coverage of brain endothelial cells. Arterioscler Thromb Vasc Biol 2013;33:1561-7.

23. Wei LM, Zhu YQ, Bao YQ, Lu HT, Zhang PL, Zhao YW, Li M, Zhao JG. Atherosclerosis in intracranial or extracranial vessels in diabetic patients and the association with stroke subtype. Quant Imaging Med Surg 2019;9:960-7.

24. Mapoure YN, Ba H, Ayeah CM, Kenmegne C, Luma $\mathrm{HN}$, Njamnshi AK. Acute stroke patients with newly diagnosed diabetes mellitus have poorer outcomes than those with previously diagnosed diabetes mellitus. J Stroke Cerebrovasc Dis 2018;27:2327-35.

25. Dhawan SS, Avati Nanjundappa RP, Branch JR, Taylor WR, Quyyumi AA, Jo H, McDaniel MC, Suo J, Giddens D, Samady H. Shear stress and plaque development. Expert Rev Cardiovasc Ther 2010;8:545-56.

26. Caro CG, Fitz-Gerald JM, Schroter RC. Arterial wall

Cite this article as: $\mathrm{Wu} \mathrm{J}$, Wang $\mathrm{P}$, Zhou L, Zhang D, Chen Q, Mao C, Su W, Huo Y, Peng J, Yin X, Chen G. Hemodynamics derived from computational fluid dynamics based on magnetic resonance angiography is associated with functional outcomes in atherosclerotic middle cerebral artery stenosis. Quant Imaging Med Surg 2022;12(1):688-698. doi: 10.21037/qims-21-337 shear and distribution of early atheroma in man. Nature 1969;223:1159-60.

27. Leng X, Wong KS, Liebeskind DS. Evaluating intracranial atherosclerosis rather than intracranial stenosis. Stroke 2014;45:645-51.

28. Samady H, Eshtehardi P, McDaniel MC, Suo J, Dhawan SS, Maynard C, Timmins LH, Quyyumi AA, Giddens DP. Coronary artery wall shear stress is associated with progression and transformation of atherosclerotic plaque and arterial remodeling in patients with coronary artery disease. Circulation 2011;124:779-88.

29. Liu D, Scalzo F, Rao NM, Hinman JD, Kim D, Ali LK, Saver JL, Sun W, Dai Q, Liu X, Liebeskind DS. Fluidattenuated inversion recovery vascular hyperintensity topography, novel imaging marker for revascularization in middle cerebral artery occlusion. Stroke 2016;47:2763-9.

30. Zhai DY, Zhu SG, Zhang W, Li X, Zhu YL. Infarct morphology assessment in patients with carotid artery/ middle cerebral artery occlusion using fast fluid-attenuated inversion recovery (FLAIR) vascular hyperintensity (FVH). PLoS One 2017;12:e0188078.

31. Samuels OB, Joseph GJ, Lynn MJ, Smith HA, Chimowitz MI. A standardized method for measuring intracranial arterial stenosis. AJNR Am J Neuroradiol 2000;21:643-6.

32. Furie KL, Kasner SE, Adams RJ, Albers GW, Bush RL, Fagan SC, Halperin JL, Johnston SC, Katzan I, Kernan WN, Mitchell PH, Ovbiagele B, Palesch YY, Sacco RL, Schwamm LH, Wassertheil-Smoller S, Turan TN, Wentworth D; American Heart Association Stroke Council, Council on Cardiovascular Nursing, Council on Clinical Cardiology, and Interdisciplinary Council on Quality of Care and Outcomes Research. Guidelines for the prevention of stroke in patients with stroke or transient ischemic attack: a guideline for healthcare professionals from the american heart association/american stroke association. Stroke 2011;42:227-76. 